\title{
Controlling Mouse using Hand Gesture Recognition
}

\author{
Shivanku Mahna \\ Amity School of Engineering \\ and Technology \\ Sector 125, Noida, UP
}

\author{
Ketan Sethi \\ Delhi Technological University \\ Shahbad Daulatpur, Main \\ Bawana Road, New Delhi
}

\author{
Sravan Ch \\ Amity School of Engineering \\ and Technology \\ Sector 125, Noida, UP
}

\begin{abstract}
Humans communicate with one another not only through their vocal abilities but also through the gestures that they make. A gesture can go a great way in putting through our point and making the other person understand us and computers are no different in this and can be controlled and made to respond to hand gestures using a gesture recognition system.

The primary goal of a gesture recognition system is to identify specific human gesture and use the gesture for controlling the device in the manner specified for that gesture in the gesture recognition system. By implementing real time gesture recognition system, a user can control a computer by simply doing gestures in front of the web cam which is attached with the laptop/computer. And to implement this system, we have used real-time hand tracking algorithm, extraction algorithm and feature extraction.
\end{abstract}

\section{General Terms}

Gesture Recognition, Gesture Recognition System (GRS), Hand Tracking, Feature Extraction, Background Subtraction.

\section{Keywords}

Gesture Recognition System- GRS

\section{INTRODUCTION}

Using gestures for controlling devices is a technology which yet to be exploited as much as other technologies have been exploited but it is surely something to lookout for and the technology for future and hence keeping this in mind, we are making this research paper so that we can make it more clear to the readers that how exactly this technology can be put to use and what all aspects and algorithms are required to do so. So the logic behind developing a gesture recognitions system is to create a system which can identify specific human gestures and use them to either convey some information to the system or to control the device itself and make it perform a certain action in correspondence to a certain gesture. And to do so, we will build a GRS to control a mouse using human gestures. But to understand this technology we firstly need to understand what gesture exactly is.

Gesture is the movement of body or a part of body to convey a meaning to the other person. The movement can be in conjunction with the verbal message or can be made to show disagreement with what is being spoken. But how can we use a gesture and encode some information in it? What is the system architecture and the concepts required for implementing a GRS? The answers to all the above questions will be in the sections to follow. We have also covered, through the course of this paper, some really important aspects of a gesture recognition system like :- what are the major issues involved in implementing a gesture recognition system and last but certainly the most important thing to lookout for, that is, the future scope of GRS.

\section{PROBLEM BEING ADDRESSED}

Mouse of a computer is a very useful object and helps in a huge way in letting us communicate with the computer. But the problem arises when the person using the mouse is a physically handicapped person. So while in some cases it can be difficult to use a mouse, in some other it might be almost impossible due to the extent of being handicapped. So to address this issue we have designed a system that will help in controlling the mouse with the help of a GRS. Another problem that will be addressed while making GRS is that usage of mouse is inconvenient while travelling and so a better system needs to be in place to help for the same and GRS while come to our rescue.

\section{WORKING OF GESTURE RECOGNITION SYSTEM}

In this paper we require to establish a relation between hand gesture and a hardware system using a set of codes which could be implemented using a software called "Matlab". In Matlab we will integrate web cam to read hand signals and then we will use the detection codes, frame by frame, to process those signals further controlling hardware such as Mouse or even Traffic lights

\subsection{Hardware Being Used}

Web Cam -Web cam is the most crucial element in hand gesture recognition system because it is interfacing hand signals with Matlab software. The working of the GRS is as shown below in Fig1 in the form of a flow chart :-

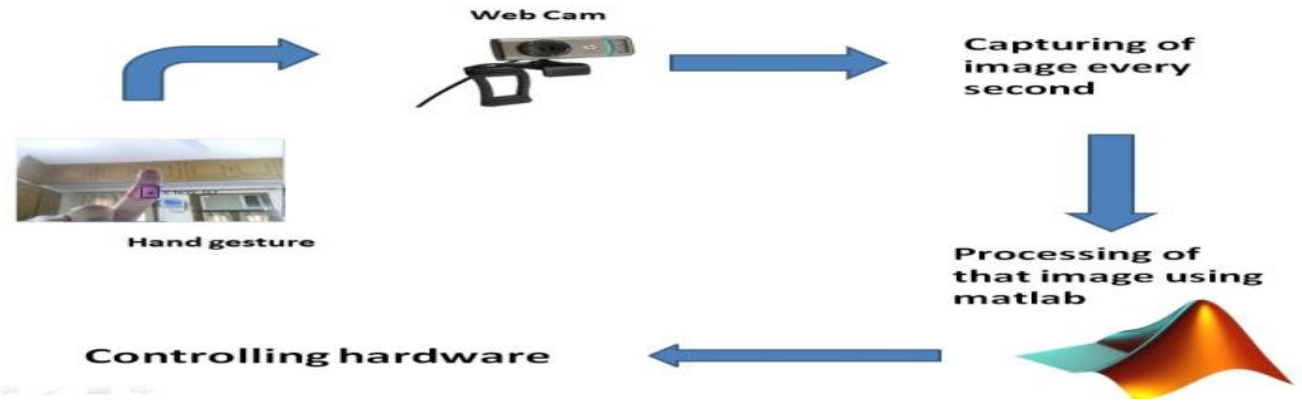

Fig 1: The web cam we used was HP-101 Specs-YUY2_320x240 


\subsection{Software Being Used}

\section{Matlab - R2012b (Version 8.0)}

To develop this GRS, we are required to write a program that can read hand gestures .Lets break this down to basic steps .Our first step will be to store the video in a variable. Our second step will be then running an infinite loop to detect gesture in every frame being performed thus detecting the gestures in the video [3].

\section{UTILIZATION OF SOFTWARE AND HARDWARE}

The next step is controlling the actual hardware. This can be achieved by integrating the microcontroller with Matlab and writing a series of conditional commands to operate it.

We will be performing two important steps with the commands that we shall write. The first one will be written for detecting the color and the second one shall be for controlling the mouse through the hand gestures. A detailed set of commands is written below and every command is followed by a comment that explains the working and function that each step shall perform.

\subsection{Commands to Perform Colour Detection are as follows}

$a=$ imread('rgb.jpg');

$$
\begin{aligned}
& \% \text { (this command reads image 'rgb.jpg' in } \\
& \text { variable 'a'.) } \\
& b=i m 2 b w(a) \text {; } \\
& \%(\text { conversion of coloured image to black } \\
& \text { and white.) } \\
& R=a(:, ., 1) \text {; } \\
& \% \text { (converts image in gray scale with red } \\
& \quad \text { component value nearer to ' } 1 \text { ' rest closer } \\
& \text { to ' } 0 \text { ' }) \\
& G=a(:, ., 2) \text {; } \\
& \% \text { (converts image in gray scale with green } \\
& \text { component value nearer to ' } 1 \text { ' rest closer } \\
& \text { to ' } 0 \text { ' })
\end{aligned}
$$

$B=a(:,:, 3)$;

$\%$ (converts image in gray scale with blue component value nearer to ' 1 ' rest closer to ' 0 ' $\%$ )

$R e=i m 2 b w(R, 180 / 255)$;

$\%$ (converts the grayscale image to black \& white with threshold (.70) i.e converting all pixels with value more than 0.7 to ' 1 ' and anything lower to that to ' 0 ')

$G e=i m 2 b w(G, 150 / 255)$;

$\%$ (converts the grayscale image to black \& white with threshold (.580) i.e converting all pixels with value more than 0.58 to ' 1 ' and

anything lower to that to ' 0 ')

$B e=\operatorname{im} 2 b w(B, 150 / 255)$;

$\%$ (converts the gray scale image to black \& white with threshold (.580) i . e converting all pixels with value more than 0.58 to ' 1 ' and anything lower to that to ' 0 ')

$r e d=(R e-G e)-B e$;

\%subtraction of images

subplot(4,3,(1:2));imshow(a);

subplot(4,3,3);imshow(b);

$\operatorname{subplot}(4,3,4) ; \operatorname{imshow}(R)$;

subplot(4,3,5);imshow $(G)$;

subplot(4,3,6);imshow(B);

subplot(4,3,7);imshow(Re);

subplot(4,3,8);imshow $(\mathrm{Ge})$;

subplot(4,3,9);imshow(Be);

subplot(4,3,10);imshow(red);

subplot(4,3,12);imshow (blue);

$\%$ plotting of images in a matrix

Output

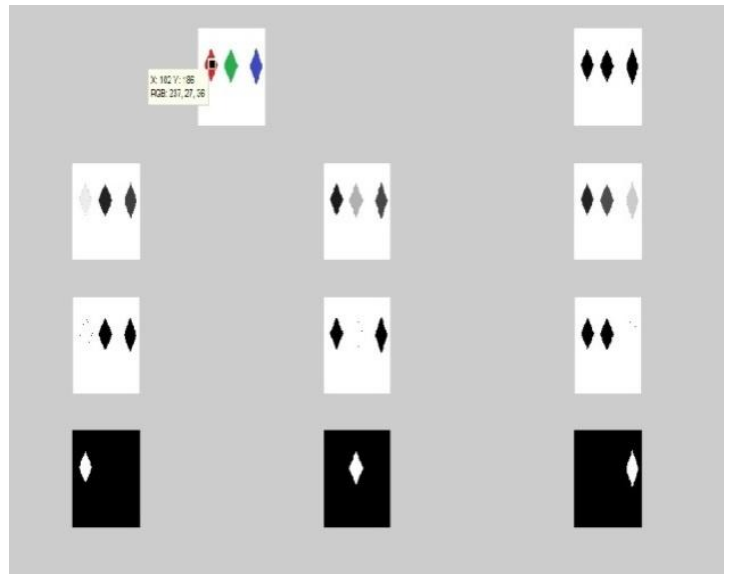

\subsection{Commands to Control the Mouse are as follows}

v=videoinput('winvideo' ,2,'YUY2_320x240');

$\%$ web cam data is stored in variable

'v'

set(v,'TriggerRepeat'

,inf);

$\%$ match camera frame rate

and processing speed

$\operatorname{start}(\mathrm{v})$;

$\%$ starts image aquisition 
import java.awt.Robot;

$\%$ this command is used if

the mouse is to be controlled according to the program

import java.awt.event.*;

mouse $=$ Robot;

mouse.mouseMove $(0,0)$;

while $(1>0)$

$\mathrm{n}=$ getsnapshot $(\mathrm{v})$;

$\mathrm{c}=\operatorname{rgb} 2 \operatorname{gray}(\mathrm{b})$;

$\mathrm{r}=\mathrm{b}(:,:, 1)$;

$\mathrm{c}=$ imsubtract $(\mathrm{r}, \mathrm{c})$;

$\mathrm{c}=\mathrm{im} 2 \mathrm{bw}(\mathrm{c}, 0.18)$;

bwareaopen $(c, 300)$;

$\%$ this is an infinite loop which reads frame every second and detects no. Of red objects in the frame thus due to looping red objects are detected in the video

$\mathrm{c}=\mathrm{bwlabel}(\mathrm{c})$;

stats=regionprops $(\mathrm{c}$, 'Centroid')

figure(1);imshow(c);

$\%$ the while $(1>0)$ is an

hold on

flushdata(v);

if(length(stats)==1)

$\%$ if 1 red object the following loop is run

for object=1:length(stats)

$\mathrm{bc}=$ stats(object).Centroid;

$b c(1)=(1366 / 1024) * b c(1)$; $b c(2)=(768 / 1024) * b c(2)$;

mouse.mouseMove(bc(1),bc(2));

end

$\%$ in this loop bc(1) is

' $\mathrm{x}$ ' and bc(2) is ' $\mathrm{y}$ '

As we initialised mouse $(\mathrm{x}, \mathrm{y})$ to 0,0 so now mouse.mouseMove(bc(1), bc(2)) moves the cursor where the red object is moved

else

if (length(stats)==2)

$\%$ when 2 red obects are detected left click of mouse is pressed

mouse.mousePress(InputEvent.BUTTON1_MASK) ;

else

mouse.mouseRelease(InputEvent.BUTTON1_MASK);

end

if(length(stats)==3)

$\%$ when 3 red obects are detected right click of mouse is pressed

mouse.mousePress(InputEvent.BUTTON3_MASK) ;

else

mouse.mouseRelease(Input Event.BUTTON3_MASK);

end

end

end

\section{WORKING IN MATLAB}

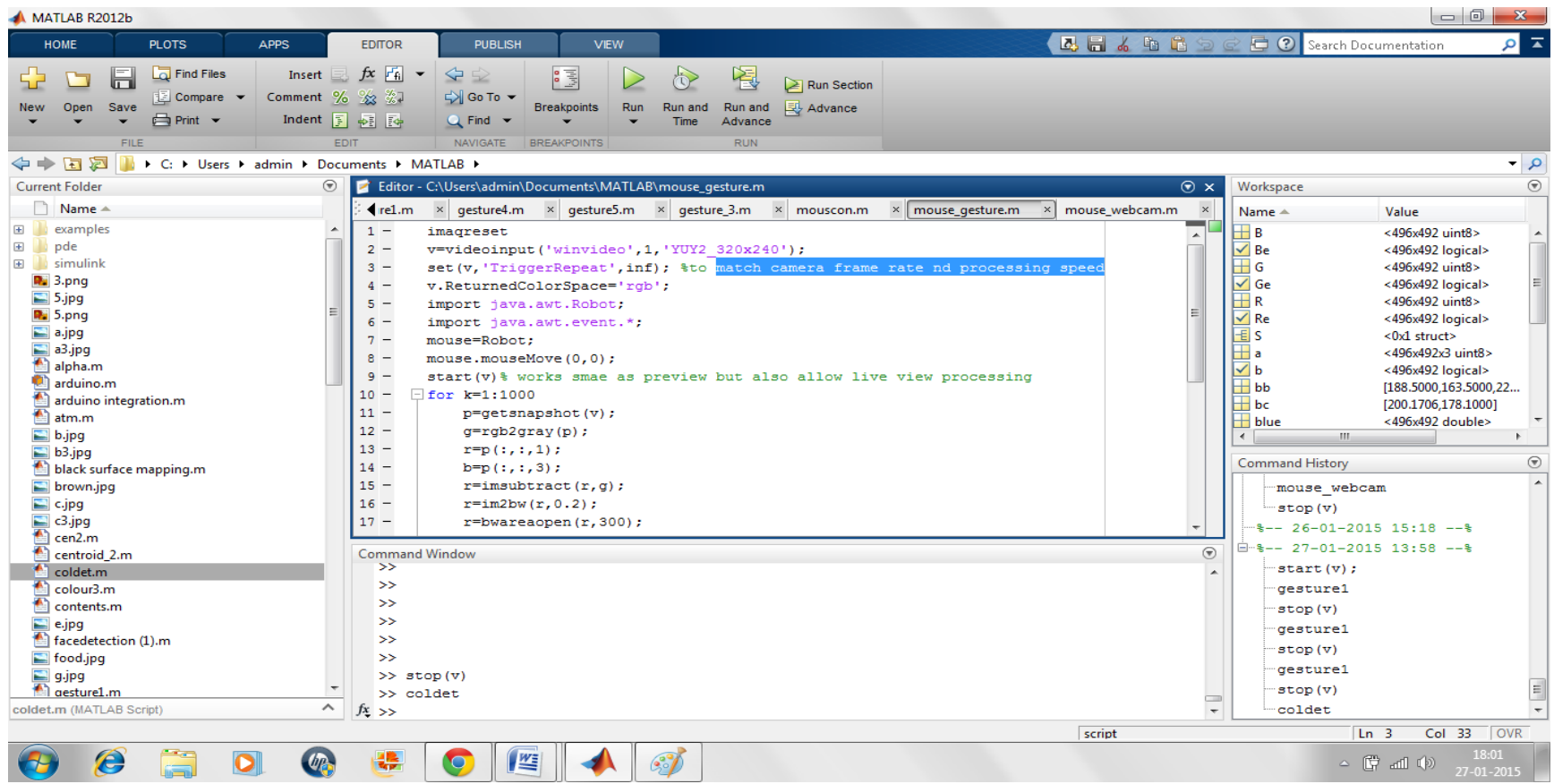

Fig2. Programs written in the editor window 
The above mentioned programs are written in the editor window and the run option is selected. After the run option is selected, the web cam turns on and starts detecting objects. Since the above set of command have been written for the detection of red objects, the web cam shall start detecting red objects as shown in the following image. Since the algorithm is for the detection of red color, so to control the mouse, one should wrap red bands around 3 fingers [1]. The functioning shall happen as follows:
- When two fingers are detected, the left key (left click) is pressed.

- When three fingers are detected, right key(right click) is pressed.

So in this way a mouse can be controlled using pattern recognition coding, Matlab and a web cam catering to the people who cannot use a mouse.

- When one finger is detected, the cursor moves where the finger moves.

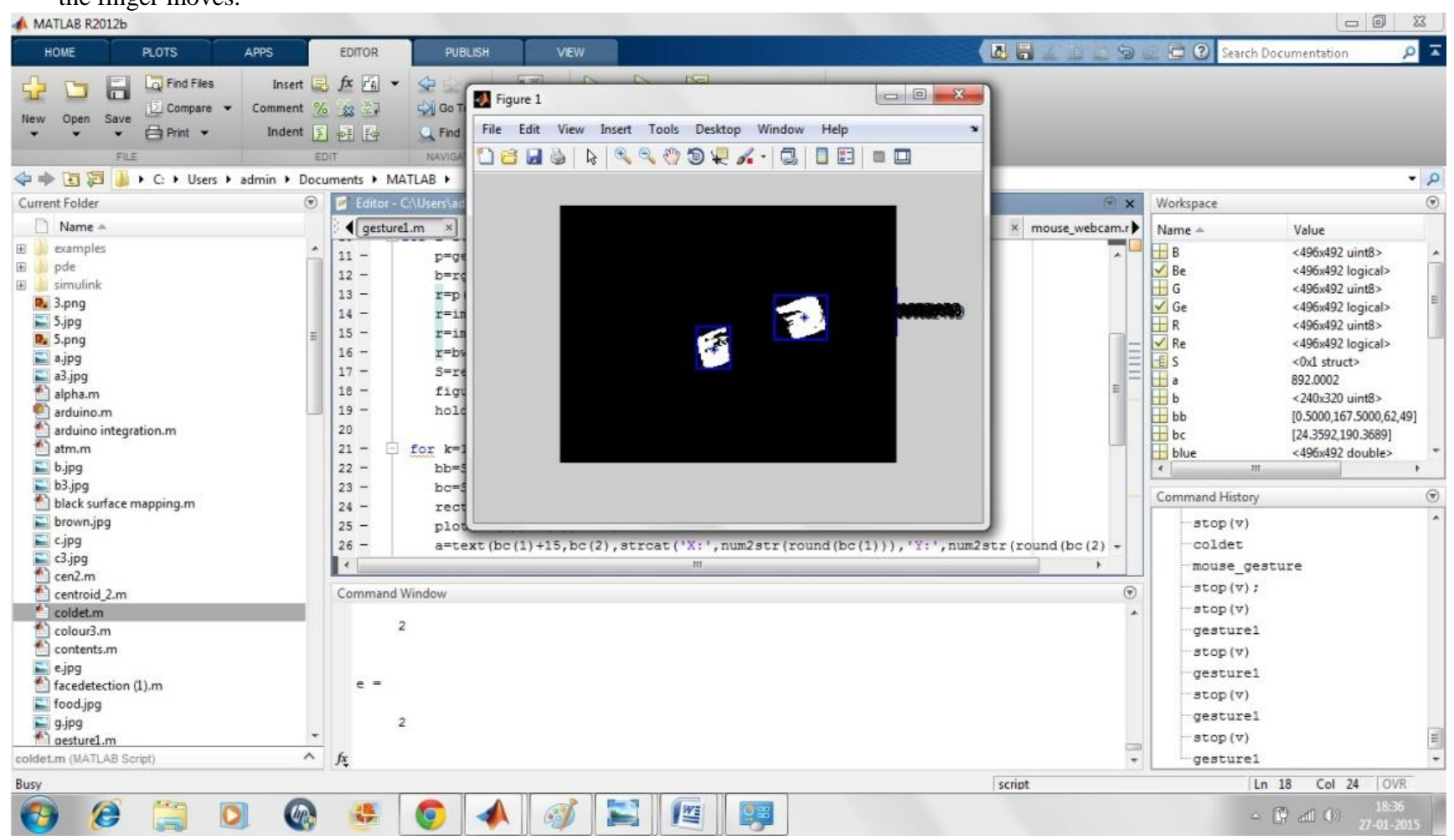

Fig 3. Output screen showing detection of two fingers

\section{APPLICATIONS AND FUTURE ENHANCEMENT \\ 6.1 Applications}

- The hand gesture recognition can be utilized for movement of the cursor in computers without touching the mouse as shown in this paper.

- The same concept can be used in object detection which would lead to many applications such as the self driving car or traffic light control depending on the no. of cars. This forms the major base for the vast area of applications called the artificial intelligence [2].

\subsection{Future Enhancement}

Artificial Intelligence will constitute a major part of our future. By every innovation, we are trying to reduce the effort of humans and to reduce the effort. Artificial Intelligence is going to be very important and play a very vital role.

- Driverless cars will be seen in very near future.

- Face detection which can only be seen in high end phones but will become common in even low budget phones in future.

\section{CONCLUSION}

We have successfully presented with a method of using a mouse that can be used by handicapped as well as non handicapped people. The method is very efficient and does not require anything besides the red bands and a web cam.

\section{ACKNOWLEDGEMENT}

We would like to express our gratitude towards a number of people whose unconditional support and considerations have been an invaluable asset during the course of this research work.

\section{REFERENCES}

[1] Hand gesture recognition using a real-time tracking method and hidden Markov model sq Feng-Sheng Chen, Chih-Ming Fu, National Tsing Hua University, ROC Received 15 January 2001; received in revised form 2 January 2003; accepted 20 March 2003.

[2] The MathWorks, Inc.), in 'Digital Image Processing Using MATLAB' Second Edition,2009 by Gatesmark, LLC.

[3] Alasdair McAndrew, in 'An Introduction to Digital Image Processing with Matlab, Notes for SCM2511 Image Processing 1', School of Computer Science and Mathematics, Victoria University of Technology 